\title{
Minimax Lower Bounds for the Two-Armed Bandit Problem*
}

\author{
Sanjeev R. Kulkarni \\ Department of Electrical Engineering \\ Princeton University, Princeton, N.J., 08544 \\ kulkarni@ee.princeton.edu \\ Gábor Lugosi \\ Department of Economics, \\ Pompeu Fabra University, \\ Ramon Trias Fargas 25-27, 08005 Barcelona, Spain. \\ lugosi@upf.es
}

Feb. 21, 1997

\begin{abstract}
We obtain minimax lower bounds on the regret for the classical two-armed bandit problem. We provide a finite-sample minimax version of the well-known $\log n$ asymptotic lower bound of Lai and Robbins. Also, in contrast to the $\log n$ asymptotic results on the regret, we show that the minimax regret is achieved by mere random guessing under fairly mild conditions on the set of allowable configurations of the two arms. That is, we show that for every allocation rule and for every $n$, there is a configuration such that the regret at time $n$ is at least $1-\epsilon$ times the regret of random guessing, where $\epsilon$ is any small positive constant.
\end{abstract}

${ }^{*}$ This work was supported in part by the National Science Foundation under NYI grant IRI-9457645. 


\section{Introduction}

In the classical two-armed bandit problem (originating from the work in $[8,9]$ ), there are two unknown distributions $P_{1}$ and $P_{2}$ associated with arm 1 and arm 2, respectively. At each time we are allowed to select an arm from which to receive a reward drawn according to the distribution for that arm. Our goal is to maximize the expected sum of the rewards. Let $m_{1}$ and $m_{2}$ denote the expected values corresponding to $P_{1}$ and $P_{2}$, respectively. If we knew which one of $m_{1}$ and $m_{2}$ is larger, we could keep selecting the arm with larger mean, and after time $n$, our expected reward would be $n \max \left(m_{1}, m_{2}\right)$. Since the distributions $P_{1}$ and $P_{2}$ are unknown, the expected reward will always be smaller than this optimal value. The difference between $n \max \left(m_{1}, m_{2}\right)$ and the expected reward is called the regret. Note that if, in each step, we select an arm independently with equal probabilities, the regret is $n \Delta / 2$, where $\Delta=\left|m_{1}-m_{2}\right|$. The results of Lai and Robbins [7] and subsequent extensions by others (e.g., [1, 2, 3, 4]) showed that in a fairly strong asymptotic sense the optimum achievable regret is $\Delta \log n / I$, where $I$ is the Kullback-Leibler divergence between $P_{1}$ and $P_{2}$. In this paper, we consider the problem from a non-asymptotic minimax perspective. We offer a finite-sample minimax version of the Lai-Robbins lower bound (see Theorem 1 below). This result can be used to provide bounds on the sample size necessary to guarantee a desired performance. Also, in sharp contrast to the well-known $\log n$ asymptotic results on the regret, we show that the minimax regret is about $n \Delta / 2$ under fairly mild conditions on the set of allowable configurations of the two arms. We show that if the set of allowable configurations is sufficiently "large," then for any $n$, for any small $\epsilon$, and for any strategy of selecting arms, there is a configuration such that the regret is larger than $(1-\epsilon) n \Delta / 2$. In other words, regardless of how large $n$ is, up to time $n$, the "bad" arm will be played almost half of the time for some configuration. That is, in the minimax sense, no arm-selection strategy can perform better than completely random selections.

\section{Formulation and Lower Bounds}

Let a configuration $\Theta=\left(\theta_{1}, \theta_{2}\right)$ be a pair of parameters determining the distributions of the two arms. That is, if arm $i$ is pulled, a reward is payed according to the probability density $f_{\theta_{i}}(i=1,2)$. All densities are understood with respect to a common dominating $\sigma$-finite measure $\lambda$ on the real line. Denote the respective means by

$$
m_{i}=\int x f_{\theta_{i}}(x) d \lambda(x), \quad i=1,2
$$

and let $\Delta=\left|m_{1}-m_{2}\right|$. Assume without loss of generality that $m_{1}>m_{2}$, that is, arm 1 is optimal. We denote the measure and expectation with respect to the distribution associated with $\theta$ by $P_{\theta}$ and $E_{\theta}$, respectively.

Introduce the alternative configuration $\Theta^{\prime}=\left(\theta_{1}^{\prime}, \theta_{2}\right)$, for some $\theta_{1}^{\prime}$ such that $m_{1}^{\prime}=$ $\int x f_{\theta_{1}^{\prime}}(x) d \lambda(x)=m_{2}-\Delta$. Thus, the distribution of the reward after pulling arm 2 is unchanged, but arm 2 is optimal in configuration $\Theta^{\prime}$. 
Our bounds involve the information divergence (or Kullback-Leibler number) between the densities $f_{\theta_{1}}$ and $f_{\theta_{1}^{\prime}}$ given by

$$
I=I\left(\theta_{1}^{\prime}, \theta_{1}\right)=\int f_{\theta_{1}^{\prime}}(x) \log \left(\frac{f_{\theta_{1}^{\prime}}(x)}{f_{\theta_{1}}(x)}\right) d \lambda(x)=E_{\theta^{\prime}}\left\{\log \left(\frac{f_{\theta_{1}^{\prime}}(x)}{f_{\theta_{1}}(x)}\right)\right\},
$$

as well as a variance-like quantity, denoted $V$, related to the information divergence $I$ by

$$
V=\int f_{\theta_{1}^{\prime}}(x) \log ^{2}\left(\frac{f_{\theta_{1}^{\prime}}(x)}{f_{\theta_{1}}(x)}\right) d \lambda(x)-I^{2}=E_{\theta^{\prime}}\left\{\log ^{2}\left(\frac{f_{\theta_{1}^{\prime}}(x)}{f_{\theta_{1}}(x)}\right)\right\}-I^{2} .
$$

(All logarithms are of the natural base.)

An adaptive allocation rule $\Phi=\left(\phi_{1}, \phi_{2}, \ldots\right)$ is a sequence of random variables taking values in $\{1,2\}$ such that $\phi_{j}$ is measurable with respect to the $\sigma$-field $\mathcal{F}_{i-1}$ generated by the previous values $\phi_{1}, X_{1}, \ldots, \phi_{i-1}, X_{i-1}$, where $X_{1}, X_{2}, \ldots$ are the random variables denoting the sequence of rewards obtained. That is, based on the previous rewards $\left(X_{1}, \ldots, X_{i-1}\right)$ and the previous selections $\left(\phi_{1}, \ldots, \phi_{i-1}\right), \phi_{i}$ denotes whether arm 1 or arm 2 is to be pulled at time $i$. Under a particular adaptive allocation rule and configuration $\Theta$, our reward up to and including time $n$ is

$$
S_{n}=\sum_{i=1}^{n} X_{i}
$$

Since $E\left[X_{i} \mid \mathcal{F}_{i-1}\right]=m_{\phi_{i}}$, the expected reward is

$$
E\left[S_{n}\right]=\sum_{i=1}^{n} E\left[X_{i}\right]=\sum_{i=1}^{n} E\left(E\left[X_{i} \mid \mathcal{F}_{i-1}\right]\right)=E\left[\sum_{i=1}^{n} m_{\phi_{i}}\right] .
$$

and the regret at time $n$ is

$$
R_{n}(\Theta)=n \max \left(m_{1}, m_{2}\right)-E\left[S_{n}\right] .
$$

In other words, $R_{n}(\Theta)$ is $\Delta$ times the expected number of times the arm with worse expected payoff is pulled.

Our goal is to obtain lower bounds on the minimum value of

$$
\max \left(R_{n}(\Theta), R_{n}\left(\Theta^{\prime}\right)\right)
$$

over all possible adaptive allocation rules. For all integers $n>0$ and $\alpha>c$, introduce

$$
\Lambda_{\alpha, n}=e^{-\alpha I}\left(1-p_{\alpha, n}\right),
$$

where

$$
p_{\alpha, n}=P_{\theta^{\prime}}\left\{\sum_{i=1}^{n} \log \left(\frac{f_{\theta_{1}}\left(x_{i}\right)}{f_{\theta_{1}^{\prime}}\left(x_{i}\right)}\right) \leq-\alpha I\right\}
$$

Theorem 1 For any $n, a_{n} \in(0,1), c_{n} \in(0, n), \alpha>c_{n}$, and for any adaptive allocation rule,

$$
\max \left(R_{n}(\Theta), R_{n}\left(\Theta^{\prime}\right)\right) \geq \Delta \min \left(c_{n}\left(1-a_{n}\right),\left(n-c_{n}\right) a_{n} \Lambda_{\alpha, c_{n}}\right) .
$$


To interpret the theorem, we need lower bounds for $\Lambda_{\alpha, n}$, that is, upper bounds for $p_{\alpha, n}$. Note that $p_{\alpha, n}$ is the probability that the sum of $n$ i.i.d. random variables (with negative mean $-I)$ is less than the mean of the sum minus $(\alpha-n) I$. Thus, it follows by Chebyshev's inequality that

$$
\Lambda_{\alpha, n} \geq e^{-\alpha I}\left(1-\frac{n V}{(\alpha-n)^{2} I^{2}}\right) .
$$

We will see that (1) is a satisfactory bound if the ratio $V / I$ is not too large. This is indeed the case for many interesting cases. The next two examples serve as illustration.

Example. Let $f_{\theta_{1}}$ be the normal density with mean $m_{1}$ and variance $\sigma^{2}$, and let $f_{\theta_{1}^{\prime}}$ be the normal density with mean $m_{1}^{\prime}$ and variance $\sigma^{2}$. Then straightforward calculation shows that $V=I$ for all values of $m_{1}, m_{1}^{\prime}$, and $\sigma$.

Example. Let $\theta_{1}$ correspond to the Bernoulli distribution $P_{\theta_{1}}(\{0\})=p, P_{\theta_{1}}(\{1\})=1-p$, and let $\theta_{1}^{\prime}$ be defined by $P_{\theta_{1}^{\prime}}(\{0\})=1-p, P_{\theta_{1}^{\prime}}(\{1\})=p$ and assume that $p>1 / 2$. Then using the inequality $\log x \leq x-1$,

$$
\frac{V}{I}=\frac{\left(1-(2 p-1)^{2}\right) \log ^{2}(p /(1-p)}{(2 p-1) \log (p /(1-p)} \leq 4 p \leq 4 .
$$

Note that in this example we can take $f_{\theta_{2}}$ to be any density with mean $m_{2}=1 / 2$, for example, we may let $P_{\theta_{2}}(\{1 / 2\})=1$.

In specific situations, one may get much sharper estimates. For example, if both $f_{\theta_{1}}$ and $f_{\theta_{1}^{\prime}}$ are Gaussian with variance $\sigma^{2}$, then $\log \left(f_{\theta_{1}}(X) / f_{\theta_{1}^{\prime}}(X)\right)$ also has a Gaussian distribution, so one may get sharper estimates for $p_{\alpha, n}$ by using standard bounds for the tail of a Gaussian distribution, but we do not detail these, rather straightforward, bounds here.

Corollary 1 Fix any $\epsilon \in(0,1)$. If $n$ is so large that

$$
n^{\epsilon^{2}} \geq \max \left(4 \frac{(1-\epsilon)^{2}}{\epsilon} \frac{\log n}{I}, e^{2 V /(I(1-\epsilon))}\right),
$$

then

$$
\max \left(R_{n}(\Theta), R_{n}\left(\Theta^{\prime}\right)\right) \geq \Delta(1-\epsilon)^{2} \frac{\log n}{I}
$$

Proof. In Theorem 1 take $a_{n}=\epsilon, c_{n}=(1-\epsilon) \log n / I$, and $\alpha=(1+\epsilon) c_{n}$. Then (1) and a straightforward calculation shows that

$$
c_{n}\left(1-a_{n}\right) \leq\left(n-c_{n}\right) a_{n} \Lambda_{\alpha, c_{n}}
$$

whenever the condition for $n$ is satisfied, and therefore $\max \left(R_{n}(\Theta), R_{n}\left(\Theta^{\prime}\right)\right) \geq c_{n}\left(1-a_{n}\right)$.

For smaller values of $n$, Theorem 1 may be used to derive much larger lower bounds: 
Corollary 2 Let $a \in(0,1)$. If $n^{1-a} \geq 4 e$ and $n^{a} I+\sqrt{n^{a} V / 2} \leq 1$ then

$$
\max \left(R_{n}(\Theta), R_{n}\left(\Theta^{\prime}\right)\right) \geq \frac{n^{a} \triangle}{2}
$$

Proof. Take $c_{n}=n^{a}, a_{n}=1 / 2$, and $\alpha=n+\sqrt{n V /\left(2 I^{2}\right)}$ in Theorem 1 .

Taking $c_{n}=n / 2$ and $a_{n}=1 / 2$ in Theorem 1 we obtain the lower bound $(n \Delta / 4) \Lambda_{\alpha, n / 2}$. The following theorem improves on this:

Theorem 2 For any $n, \alpha>n$, and for any adaptive allocation rule,

$$
\max \left(R_{n}(\Theta), R_{n}\left(\Theta^{\prime}\right)\right) \geq \frac{n \Delta}{2} \Lambda_{\alpha, n}
$$

Corollary 3 For any $n \leq\lceil 1 / I\rceil$ and for any adaptive allocation rule,

$$
\max \left(R_{n}(\Theta), R_{n}\left(\Theta^{\prime}\right)\right) \geq \frac{\Delta n}{4} e^{-1-\sqrt{V /(2 I)}} .
$$

Proof. Note that if we take $\alpha=n+\sqrt{n V /\left(2 I^{2}\right)}$,

$$
1-\frac{n V}{(\alpha-n)^{2} I^{2}}=\frac{1}{2}
$$

and therefore, the corollary follows by applying Theorem 2 with (1) for $n=\lceil 1 / I\rceil$.

Corollary 4 Let $\epsilon>0$ be arbitrary. If there exists an $\alpha>n$ such that $\alpha \leq-\log (1-$ $\epsilon) / 2 I$ and $(\alpha-n)^{2} / n \geq 2 V /\left(\epsilon I^{2}\right)$, then

$$
\max \left(R_{n}(\Theta), R_{n}\left(\Theta^{\prime}\right)\right) \geq \frac{\Delta n}{2}(1-\epsilon) .
$$

Proof. Straightforward calculation shows that if the conditions are satisfied then $e^{-\alpha I} \geq$ $\sqrt{1-\epsilon}$ and $1-n V /\left((n-\alpha)^{2} I^{2}\right) \geq \sqrt{1-\epsilon}$, so the statement follows by Theorem 2 and the bounds of $(1)$.

Corollary 4 may be applied with arbitrary $\epsilon$ in many cases when, in the class of allowable configurations, there are pairs $\left(\theta_{1}, \theta_{1}^{\prime}\right)$ with arbitrarily small information divergence. The following two special cases illustrate such situations.

Corollary 5 Suppose $P_{2}$ is an arbitrary distribution with mean zero (which can even be known). Suppose $P_{1}$ is Gaussian with mean either $\Delta$ or $-\Delta$ with arbitrary variance. Then for every adaptive allocation rule, for every $\epsilon>0$, and for every $n$, there is a configuration such that the regret at time $n$ is at least $(1-\epsilon) n \Delta / 2$.

Corollary 6 Let $S$ be the set of all configurations $\Theta=\left(\theta_{1}, \theta_{2}\right)$ such that both $P_{\theta_{1}}$ and $P_{\theta_{2}}$ are Bernoulli distributions (i.e., of the form $P_{\theta}(\{0\})=p, P_{\theta}(\{1\})=1-p$ for some $p)$. Then for every adaptive allocation rule $\Phi$, for every $\epsilon>0$, and for every $n$,

$$
\sup _{\Theta \in S} R_{n}(\Theta) \geq \frac{n \Delta}{2}(1-\epsilon)
$$

where $\Delta$ is the difference between the means corresponding to the two arms. 


\section{A Change-of-Measure Lemma}

As before, let the vector $\mathbf{X}=\left(X_{1}, \ldots, X_{n}\right)$ of random variables denote the rewards up to time $n$ if a particular adaptive allocation rule is used. Let $\mathrm{x}=\left(x_{1}, x_{2}, \ldots, x_{n}\right) \in \mathcal{R}^{n}$ denote a fixed realization of $\mathbf{X}$.

$T_{\mathbf{x}}(1)$ and $T_{\mathbf{x}}(2)$ denote the number of times arm 1, and arm 2 are pulled up to time $n$.

The key part of the proofs of the results in the previous section is the following measure-transformation lemma, which is based on ideas of Lai and Robbins [7].

Lemma 1 For any integer $k \in[0, n]$, and $\alpha>k$,

$$
P_{\Theta}\left\{T_{\mathbf{x}}(1)=k\right\} \geq \Lambda_{\alpha, k} P_{\Theta^{\prime}}\left\{T_{\mathbf{x}}(1)=k\right\} .
$$

Proof. Let $J \subset\{1, \ldots, n\}$ be a set of indices. On $J$, introduce the likelihood ratio

$$
L_{J}(\mathbf{x})=\sum_{j \in J} \log \left(\frac{f_{\theta_{1}}\left(x_{j}\right)}{f_{\theta_{1}^{\prime}}\left(x_{j}\right)}\right) .
$$

The first step of the proof is trivial:

$$
P_{\Theta}\left\{T_{\mathbf{x}}(1)=k\right\} \geq P_{\Theta}\left\{T_{\mathbf{x}}(1)=k, L_{B(\mathbf{x})}(\mathbf{x})>-\alpha I\right\}
$$

where $B(\mathbf{x})$ is the set of indices indicating the times when arm 1 is pulled by the allocation rule based on the sequence of observations $\mathbf{x}$.

For each index set $J$, define $A_{J} \subset \mathcal{R}^{n}$ by

$$
A_{J}=\left\{\mathbf{x}: B(\mathbf{x})=J, L_{B(\mathrm{x})}(\mathbf{x})>-\alpha I\right\} .
$$

Thus,

$$
P_{\Theta}\left\{T_{\mathbf{x}}(1)=k, L_{B(\mathbf{x})}(\mathbf{x})>-\alpha I\right\}=P_{\Theta}\left\{\bigcup_{J:|J|=k} A_{J}\right\}=\sum_{J:|J|=k} P_{\Theta}\left\{A_{J}\right\}
$$

Now

$$
\begin{aligned}
P_{\Theta}\left\{A_{J}\right\} & =\int_{A_{J}}\left(\prod_{j \in J} f_{\theta_{1}}\left(x_{j}\right)\right)\left(\prod_{j \notin J} f_{\theta_{2}}\left(x_{j}\right)\right) d \lambda\left(x_{1}\right) \cdots d \lambda\left(x_{n}\right) \\
& =\int_{A_{J}}\left(\prod_{j \in J} \frac{f_{\theta_{1}}\left(x_{j}\right)}{f_{\theta_{1}^{\prime}}\left(x_{j}\right)}\right)\left(\prod_{j \in J} f_{\theta_{1}^{\prime}}\left(x_{j}\right)\right)\left(\prod_{j \notin J} f_{\theta_{2}}\left(x_{j}\right)\right) d \lambda\left(x_{1}\right) \cdots d \lambda\left(x_{n}\right) .
\end{aligned}
$$

But for each $\mathrm{x} \in A_{J}$, we have $L_{J}(\mathrm{x})>-\alpha I$, so

$$
\left(\prod_{j \in J} \frac{f_{\theta_{1}}\left(x_{j}\right)}{f_{\theta_{1}^{\prime}}\left(x_{j}\right)}\right)>e^{-\alpha I}
$$


and therefore

$$
P_{\Theta}\left\{A_{J}\right\} \geq e^{-\alpha I} P_{\Theta^{\prime}}\left\{A_{J}\right\}
$$

It follows that

$$
\begin{aligned}
& P_{\Theta}\left\{T_{\mathbf{x}}(1)=k, L_{B(\mathbf{x})}(\mathbf{x})>-\alpha I\right\} \\
& \quad \geq e^{-\alpha I} P_{\Theta^{\prime}}\left\{T_{\mathbf{x}}(1)=k, L_{B(\mathbf{x})}(\mathbf{x})>-\alpha I\right\} \\
& \quad=e^{-\alpha I} P_{\Theta^{\prime}}\left\{T_{\mathbf{x}}(1)=k\right\} P_{\Theta^{\prime}}\left\{L_{B(\mathbf{x})}(\mathbf{x})>-\alpha I \mid T_{\mathbf{x}}(1)=k\right\}
\end{aligned}
$$

But by the definition of $p_{\alpha, k}$, we have

$$
P_{\Theta^{\prime}}\left\{L_{B(\mathrm{x})}(\mathrm{x})>-\alpha I \mid T_{\mathbf{x}}(1)=k\right\} \geq 1-p_{\alpha, k} .
$$

Summarizing (2), (3), and (4), the proof of the lemma is complete.

\section{Proofs of Theorems 1 and 2}

Proof of Theorem 1. There are two cases. If $P_{\Theta^{\prime}}\left\{T_{x}(1) \leq c_{n}\right\}<a_{n}$, then by Markov's inequality, we have

$$
E_{\Theta^{\prime}}\left[T_{x}(1)\right] \geq c_{n}\left(1-a_{n}\right) .
$$

If, on the other hand, $P_{\Theta^{\prime}}\left\{T_{x}(1) \leq c_{n}\right\} \geq a_{n}$, then

$$
\begin{aligned}
P_{\Theta}\left\{T_{x}(1) \leq c_{n}\right\} & =\sum_{k=1}^{c_{n}} P_{\Theta}\left\{T_{x}(1)=k\right\} \\
& \left.\geq \Lambda_{\alpha, c_{n}} \sum_{k=1}^{c_{n}} P_{\Theta^{\prime}}\left\{T_{x}(1)=k\right\} \quad \text { (by Lemma 1, whenever } \alpha>c_{n}\right) \\
& =\Lambda_{\alpha, c_{n}} P_{\Theta^{\prime}}\left\{T_{x}(1) \leq c_{n}\right\} \\
& \geq a_{n} \Lambda_{\alpha, c_{n}} .
\end{aligned}
$$

Thus,

$$
P_{\Theta}\left\{T_{x}(2) \leq n-c_{n}\right\}=P_{\Theta}\left\{T_{x}(1) \leq c_{n}\right\} \geq a_{n} \Lambda_{\alpha, c_{n}},
$$

and by Markov's inequality,

$$
E_{\Theta}\left[T_{x}(2)\right] \geq\left(n-c_{n}\right) a_{n} \Lambda_{\alpha, c_{n}} .
$$

Therefore,

$$
\begin{aligned}
\max \left(R_{n}(\Theta), R_{n}\left(\Theta^{\prime}\right)\right) & =\Delta \max \left(E_{\Theta}\left[T_{x}(2)\right], E_{\Theta^{\prime}}\left[T_{x}(1)\right]\right) \\
& \geq \Delta \min \left(c_{n}\left(1-a_{n}\right),\left(n-c_{n}\right) a_{n} \Lambda_{\alpha, c_{n}}\right)
\end{aligned}
$$

and the theorem is proved.

Proof of Theorem 2. After time $n$, the regret under configuration $\Theta$ is

$$
R_{n}(\Theta)=\Delta E_{\Theta}\left[T_{\mathbf{x}}(2)\right]
$$


For any $\alpha>n$, we have

$$
\begin{aligned}
\max \left(R_{n}(\Theta), R_{n}\left(\Theta^{\prime}\right)\right) & \geq \frac{R_{n}(\Theta)+R_{n}\left(\Theta^{\prime}\right)}{2} \\
& =\Delta \frac{E_{\Theta}\left[T_{\mathbf{x}}(2)\right]+E_{\Theta^{\prime}}\left[T_{\mathbf{x}}(1)\right]}{2} \\
& =\frac{\Delta}{2} \sum_{i=1}^{n}\left(P_{\Theta}\left\{T_{\mathbf{x}}(2) \geq i\right\}+P_{\Theta^{\prime}}\left\{T_{\mathbf{x}}(1) \geq i\right\}\right) \\
& \left.\geq \frac{\Delta}{2} \Lambda_{\alpha, n} \sum_{i=1}^{n}\left(P_{\Theta}\left\{T_{\mathbf{x}}(2) \geq i\right\}+P_{\Theta}\left\{T_{\mathbf{x}}(1) \geq i\right\}\right) \quad \text { (by Lemma } 1\right) \\
& =\frac{\Delta}{2} \Lambda_{\alpha, n}\left(E_{\Theta}\left[T_{\mathbf{x}}(2)\right]+E_{\Theta}\left[T_{\mathbf{x}}(1)\right]\right) \\
& =\frac{n \Delta}{2} \Lambda_{\alpha, n},
\end{aligned}
$$

and the proof is complete.

\section{References}

[1] R. Agrawal, M. Hedge, D. Teneketzis, "Asymptotically efficient adaptive allocation rules for the multiarmed bandit problem with switching cost," IEEE Trans. Automatic Control, Vol. AC-33, No. 10, pp. 899-906, 1988.

[2] R. Agrawal, D. Teneketzis, V. Anantharam, "Asymptotically efficient adaptive allocation schemes for controlled i.i.d. processes: finite parameter space," IEEE Trans. Automatic Control, Vol. AC-34, No. 3, pp. 258-266, 1989.

[3] V. Anantharam, P. Varaiya, J. Walrand, "Asymptotically efficient allocation rules for the multiarmed bandit problem with multiple plays - part I: i.i.d. rewards," IEEE Trans. Automatic Control, Vol. AC-32, No. 11, pp. 968-976, 1987.

[4] V. Anantharam, P. Varaiya, J. Walrand, "Asymptotically efficient allocation rules for the multiarmed bandit problem with multiple plays - part II: Markovian rewards," IEEE Trans. Automatic Control, Vol. AC-32, No. 11, pp. 977-982, 1987.

[5] D.A. Berry and B. Fristedt, Bandit Problems, Chapman and Hall, 1985.

[6] J.C. Gittins, Multi-armed Bandit Allocation Indices, Wiley \& Sons, 1989.

[7] T.L. Lai and H. Robbins, "Asymptotically efficient adaptive allocation rules," $A d-$ vances in Applied Mathematics, Vol. 6, pp. 4-22, 1985.

[8] H. Robbins, "Some aspects of the sequential design of experiments," Bull. Amer. Math. Soc., Vol. 58, pp. 527-535, 1952.

[9] W.R. Thompson, "On the likelihood that one unknown probability exceeds another in view of the evidence of two samples," Biometrika, Vol. 25, pp. 275-294, 1933. 